Supporting Information

for

\title{
Two Are Better than One: Bidentate Adsorbates Offer Precise Control of Interfacial Composition and Properties
}

Han Ju Lee, Andrew C. Jamison, and T. Randall Lee*

Department of Chemistry and the Texas Center for Superconductivity, University of Houston, 4800 Calhoun Road, Houston, Texas 77204-5003, United States

*Corresponding Author: trlee@uh.edu 


\section{EXPERIMENTAL SECTION}

Materials. $\quad 1,1,1,2,2,3,3,4,4,5,5,6,6,7,7,8,8$-Heptadecafluoro-16-iodohexadecane, $\quad$ 16bromo-2,5,8,11-tetraoxahexadecane, and 3.5-bis(hydroxymethyl)phenol were prepared according to procedures available in the literature. ${ }^{\mathrm{S}-\mathrm{S} 3}$ Potassium carbonate $\left(\mathrm{K}_{2} \mathrm{CO}_{3}\right)$, methanesulfonyl chloride $(\mathrm{MsCl})$, triethylamine $\left(\mathrm{Et}_{3} \mathrm{~N}\right)$, potassium thioacetate, lithium aluminum hydride $\left(\mathrm{LiAlH}_{4}\right)$, 1-bromohexadecane, octadecanethiol (C18SH), and hexadecanethiol (HDT) were purchased from Sigma-Aldrich and used as received. The solvents tetrahydrofuran (THF) and dichloromethane $\left(\mathrm{CH}_{2} \mathrm{Cl}_{2}\right)$ were purchased from Sigma-Aldrich. Hexanes, diethyl ether, acetonitrile $\left(\mathrm{CH}_{3} \mathrm{CN}\right)$, and methanol $(\mathrm{MeOH})$ were purchased from Mallinckrodt Chemicals. Anhydrous ethanol (EtOH) was purchased from Decon Labs, Inc. Distilling over calcium hydride gave dry tetrahydrofuran and dichloromethane. Silica gel for column chromatography was obtained from Sorbent Technologies.

Synthetic Procedures. The procedures used to prepare the bidentate alkanethiols (PFPDT, HDPDT, and OEGPDT) are outlined in Scheme S1 and detailed below, while those used to prepare monodentate alkanethiols (PFT and OEGT) can be found in the literature. ${ }^{\mathrm{S} 1 \mathrm{~S} 2}$

Scheme S1. Synthesis of PFPDT, HDPDT, and OEGPDT
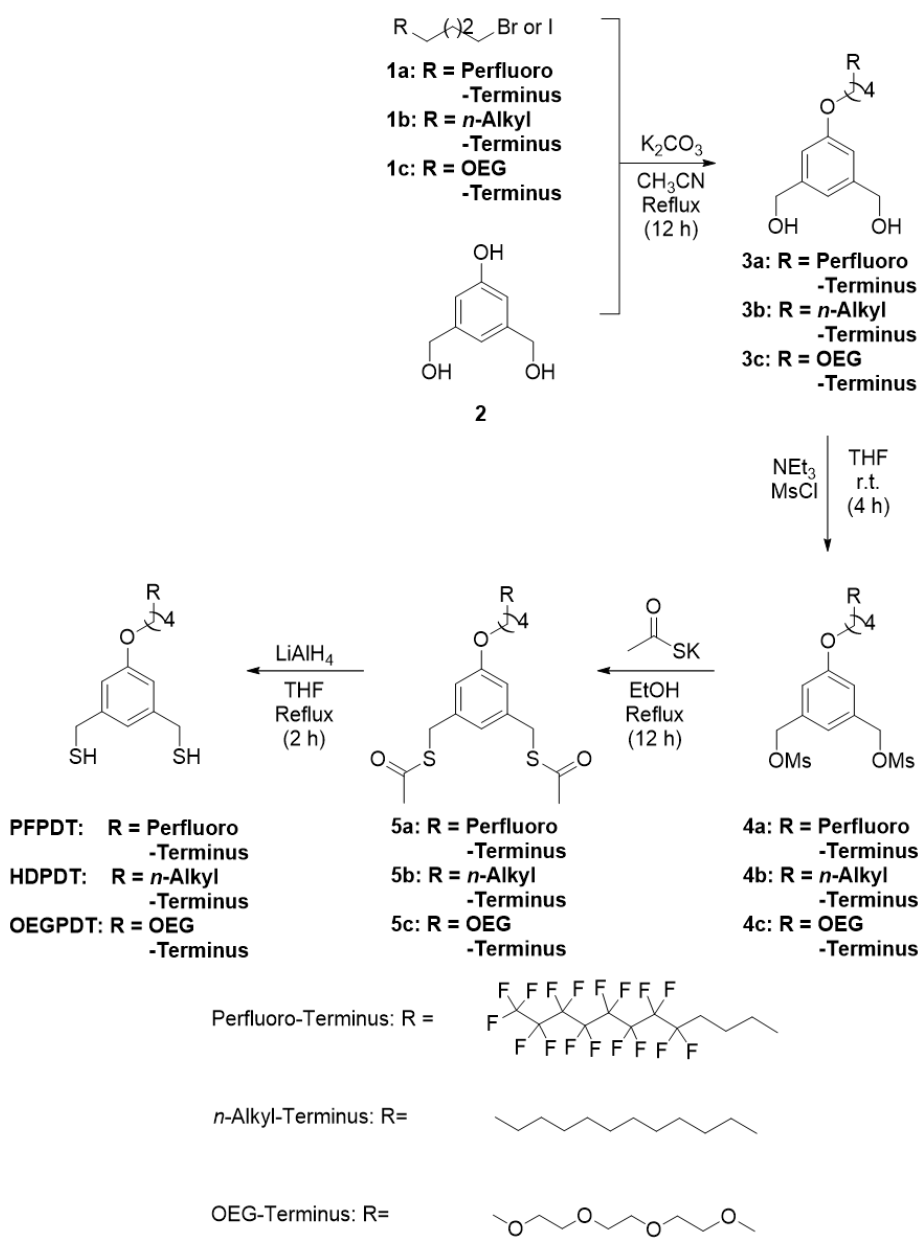
Synthesis of $\quad(5-(9,9,10,10,11,11,12,12,13,13,14,14,15,15,16,16,16$-Heptadecafluorohexadecyloxy)-1,3-phenylene)dimethanethiol (PFPDT). PFPDT was prepared according to Scheme $\mathrm{S} 1$. The experimental details are provided in the following paragraphs.

$(5-(9,9,10,10,11,11,12,12,13,13,14,14,15,15,16,16,16$-Heptadecafluorohexadecyloxy)-1,3phenylene)dimethanol (3a). A mixture of $\mathrm{K}_{2} \mathrm{CO}_{3}$ (3.76 g, $\left.20.0 \mathrm{mmol}\right)$, 3.5-bis(hydroxylmethyl)phenol (1.63 g, $10.6 \mathrm{mmol}$ ), and 1,1,1,2,2,3,3,4,4,5,5,6,6,7,7,8,8-heptadecafluoro-16iodohexadecane $(6.00 \mathrm{~g}, 9.11 \mathrm{mmol})$ in acetonitrile $(250 \mathrm{~mL})$ was refluxed for $12 \mathrm{~h}$. The resulting mixture was condensed by rotary evaporator, followed by addition of diethyl ether (200 $\mathrm{mL}$ ), and then acidification with $4.0 \mathrm{M} \mathrm{HCl}$ solution to attain a $\mathrm{pH}$ of $\sim 3$. The organic layer was washed with water $(2 \times 100 \mathrm{~mL})$, dried over $\mathrm{Na}_{2} \mathrm{SO}_{4}$, and concentrated in vacuo to produce $3.70 \mathrm{~g}(5.40 \mathrm{mmol})$ of a white solid $(59 \%$ yield $) .{ }^{1} \mathrm{H} \mathrm{NMR}\left(500 \mathrm{MHz}, \mathrm{CDCl}_{3}\right): \delta 6.92(\mathrm{~s}, 1 \mathrm{H})$, $6.84(\mathrm{~s}, 2 \mathrm{H}), 4.66(\mathrm{~s}, 4 \mathrm{H}), 3.96(\mathrm{t}, J=6.8 \mathrm{~Hz}, 2 \mathrm{H}), 2.04(\mathrm{~m}, 2 \mathrm{H}), 1.77(\mathrm{~m}, 2 \mathrm{H}), 1.63(\mathrm{~m}, 2 \mathrm{H})$, $1.36-1.46(\mathrm{~m}, 8 \mathrm{H})$.

(5-(9,9,10,10,11,11,12,12,13,13,14,14,15,15,16,16,16-Heptadecafluorohexadecyloxy)-1,3phenylene)bis(methylene) dimethanesulfonate (4a). A chosen aliquot (5.40 mmol) of (5$(9,9,10,10,11,11,12,12,13,13,14,14,15,15,16,16,16$-heptadecafluorohexadecyloxy)-1,3-

phenylene)dimethanol was introduced into a $500 \mathrm{~mL}$ round-bottomed flask containing $250 \mathrm{~mL}$ of THF and then triethylamine $(2.1 \mathrm{~mL}, 15 \mathrm{mmol})$ was added slowly to the solution. The mixture was stirred at $\mathrm{rt}$ for $30 \mathrm{~min}$. The reaction flask was placed in an ice bath, and methanesulfonyl chloride $(1.2 \mathrm{~mL}, 15 \mathrm{mmol})$ was transferred slowly to the solution while stirring. The ice bath was then removed, and the mixture was stirred for $4 \mathrm{~h}$ at $\mathrm{rt}$. After completion of the reaction, excess methanesulfonyl chloride was destroyed by adding $100 \mathrm{~mL}$ of water followed by acidification with $4.0 \mathrm{M} \mathrm{HCl}$ solution to attain a $\mathrm{pH}$ of $\sim 3$. The mixture was extracted with diethyl ether $(2 \times 150 \mathrm{~mL})$. The combined organic layers were washed with brine $(100 \mathrm{~mL})$ and water $(2 \times 100 \mathrm{~mL})$. The organic layer was dried over $\mathrm{Na}_{2} \mathrm{SO}_{4}$, filtered, and concentrated in vacuo to produce $(5-(9,9,10,10,11,11,12,12,13,13,14,14,15,15,16,16,16$-heptadecafluorohexadecyloxy)-1,3-phenylene)bis(methylene) dimethanesulfonate (3.90 g, $4.64 \mathrm{mmol})$, which was used in the next step without further purification (86\% yield). ${ }^{1} \mathrm{H} \mathrm{NMR} \mathrm{(500} \mathrm{MHz}, \mathrm{CDCl}_{3}$ ): $\delta 7.00(\mathrm{~s}, 1 \mathrm{H}), 6.94(\mathrm{~s}, 2 \mathrm{H}), 5.19(\mathrm{~s}, 4 \mathrm{H}), 3.96(\mathrm{t}, J=6.3 \mathrm{~Hz}, 2 \mathrm{H}), 2.97(\mathrm{~s}, 6 \mathrm{H}), 2.04(\mathrm{~m}, 2 \mathrm{H})$, $1.78(\mathrm{~m}, 2 \mathrm{H}), 1.60(\mathrm{~m}, 2 \mathrm{H}), 1.37-1.46(\mathrm{~m}, 8 \mathrm{H})$.

$S, S^{\prime}-(5-(9,9,10,10,11,11,12,12,13,13,14,14,15,15,16,16,16-H e p t a d e c a f l u o r o h e x a d e c y l o x y)-$ 1,3-phenylene)bis(methylene) diethanethioate (5a). A mixture of potassium thioacetate $(1.14 \mathrm{~g}$, $10.0 \mathrm{mmol})$ and $(5-(9,9,10,10,11,11,12,12,13,13,14,14,15,15,16,16,16$-heptadecafluorohexadecyloxy)-1,3-phenylene)bis(methylene) dimethanesulfonate (3.90 g, $4.64 \mathrm{mmol}$ ) in ethanol (300 $\mathrm{mL}$ ) was refluxed for $12 \mathrm{~h}$ under nitrogen. After cooling, the precipitate was filtered off, rinsed 
with ethanol, and the resulting organic phase was evaporated to dryness. The residue was taken up in a blend of diethyl ether $(100 \mathrm{~mL})$ and water $(200 \mathrm{~mL})$. The mixture was extracted with diethyl ether $(2 \times 100 \mathrm{~mL})$. The combined organic layers were washed with brine $(3 \times 100 \mathrm{~mL})$, dried over anhydrous $\mathrm{Na}_{2} \mathrm{SO}_{4}$, filtered, and concentrated by rotary evaporation, and then dried in vacuo to give $2.10 \mathrm{~g}(2.62 \mathrm{mmol})$ of $S, S^{\prime}-(5-(9,9,10,10,11,11,12,12,13,13,14,14,15,15,16,16,16-$ heptadecafluorohexadecyloxy)-1,3-phenylene)bis(methylene) diethanethioate as a pale yellow solid (56\% yield). ${ }^{1} \mathrm{H}$ NMR (500 MHz, $\left.\mathrm{CDCl}_{3}\right): \delta 6.75(\mathrm{~s}, 1 \mathrm{H}), 6.69$ (s, 2H), 4.03 (s, 4H), 3.89 $(\mathrm{t}, J=6.8 \mathrm{~Hz}, 2 \mathrm{H}), 2.34(\mathrm{~s}, 6 \mathrm{H}), 2.05(\mathrm{~m}, 2 \mathrm{H}), 1.74(\mathrm{~m}, 2 \mathrm{H}), 1.58(\mathrm{~m}, 2 \mathrm{H}), 1.35-1.44(\mathrm{~m}, 8 \mathrm{H})$.

(5-(9,9,10,10,11,11,12,12,13,13,14,14,15,15,16,16,16-Heptadecafluorohexadecyloxy)-1,3phenylene)dimethanethiol (PFPDT). To a suspension of $\mathrm{LiAlH}_{4}(0.380 \mathrm{~g}, 10.0 \mathrm{mmol})$ in THF $(300 \mathrm{~mL})$ was added a solution of $S, S^{\prime}-(5-(9,9,10,10,11,11,12,12,13,13,14,14,15,15,16,16,16-$ heptadecafluorohexadecyloxy)-1,3-phenylene)bis(methylene) diethanethioate (2.10 g, $2.62 \mathrm{mmol})$ in THF $(50 \mathrm{~mL})$ dropwise at $0{ }^{\circ} \mathrm{C}$. The ice bath was then removed, and the mixture was refluxed for $2 \mathrm{~h}$ under nitrogen. Subsequently, the reaction was quenched with degassed ethanol (25 $\mathrm{mL})$. The mixture was acidified to $\mathrm{pH} \sim 1$ by careful addition of $2.0 \mathrm{M} \mathrm{HCl}$ solution and then extracted with diethyl ether $(3 \times 100 \mathrm{~mL})$. The combined organic layers were washed with brine $(1 \times 100$ $\mathrm{mL}$ ), dried over anhydrous $\mathrm{Na}_{2} \mathrm{SO}_{4}$, filtered, and concentrated by rotary evaporation. The crude product was purified by column chromatography (hexanes:ethyl acetate $=9.7: 0.3$ ) to give $1.34 \mathrm{~g}$ $(1.87 \mathrm{mmol})$ of $(5-(9,9,10,10,11,11,12,12,13,13,14,14,15,15,16,16,16$-heptadecafluorohexadecyloxy)-1,3-phenylene)dimethanethiol as a white solid (71\% yield). ${ }^{1} \mathrm{H}$ NMR (500 $\mathrm{MHz}, \mathrm{CDCl}_{3}$ ): $\delta 6.85(\mathrm{~s}, 1 \mathrm{H}), 6.76(\mathrm{~s}, 2 \mathrm{H}), 3.95(\mathrm{t}, J=6.3 \mathrm{~Hz}, 2 \mathrm{H}), 3.68(\mathrm{~d}, J=7.4 \mathrm{~Hz}, 4 \mathrm{H}), 2.05(\mathrm{~m}, 2 \mathrm{H}), 1.78$ $(\mathrm{m}, 4 \mathrm{H}), 1.61(\mathrm{~m}, 2 \mathrm{H}), 1.38-1.47(\mathrm{~m}, 8 \mathrm{H}) .{ }^{13} \mathrm{C} \mathrm{NMR}\left(125 \mathrm{MHz}, \mathrm{CDCl}_{3}\right): \delta 159.69,143.03$, $119.93,112.94,67.97,31.09,30.91,29.32,29.27,29.23,29.13,28.98,26.08,20.15$.

Synthesis of (5-(Hexadecyloxy)-1,3-phenylene)dimethanethiol (HDPDT). HDPDT was prepared according to Scheme S1. The experimental details are provided in the following paragraphs.

(5-(Hexadecyloxy)-1,3-phenylene)dimethanol (3b). Following the procedure described for 3a, a mixture of $\mathrm{K}_{2} \mathrm{CO}_{3}(2.76 \mathrm{~g}, 20.0 \mathrm{mmol})$, bis(hydroxymethyl)phenol (1.50 g, $\left.9.74 \mathrm{mmol}\right)$ and 1-bromohexadecane $(2.80 \mathrm{~g}, 9.18 \mathrm{mmol})$ in acetonitrile $(250 \mathrm{~mL})$ was refluxed to give $3.0 \mathrm{~g}(7.9$ $\mathrm{mmol}$ ) of a white solid (86\% yield). ${ }^{1} \mathrm{H} \mathrm{NMR}\left(500 \mathrm{MHz}, \mathrm{CDCl}_{3}\right): \delta 6.24(\mathrm{~s}, 1 \mathrm{H}), 6.84(\mathrm{~s}, 2 \mathrm{H})$, $4.66(\mathrm{~s}, 4 \mathrm{H}), 3.96(\mathrm{t}, J=6.4 \mathrm{~Hz}, 2 \mathrm{H}), 1.76(\mathrm{~m}, 2 \mathrm{H}), 1.25-1.43(\mathrm{~m}, 26 \mathrm{H}), 0.87(\mathrm{t}, J=6.8 \mathrm{~Hz}, 3 \mathrm{H})$. 
(5-(Hexadecyloxy)-1,3-phenylene)bis(methylene) dimethanesulfonate (4b). Following the procedure described for $\mathbf{4 a}$, a mixture of $\mathbf{3 b}(3.0 \mathrm{~g}, 7.9 \mathrm{mmol})$ and triethylamine $(2.8 \mathrm{~mL}, 20$ $\mathrm{mmol})$ in $250 \mathrm{~mL}$ of THF was treated with methanesulfonyl chloride $(1.5 \mathrm{~mL}, 20 \mathrm{mmol})$ to give $4.1 \mathrm{~g}$ (7.6 mmol) of (5-(hexadecyloxy)-1,3-phenylene)bis(methylene) dimethanesulfonate (97\% yield). ${ }^{1} \mathrm{H} \mathrm{NMR}\left(500 \mathrm{MHz}, \mathrm{CDCl}_{3}\right): \delta 7.00(\mathrm{~s}, 1 \mathrm{H}), 6.94(\mathrm{~s}, 2 \mathrm{H}), 5.19$ (s, 4H), 3.96 (t, J = 6.3 Hz, 2H), 2.97 (s, 3H), $1.77(\mathrm{~m}, 2 \mathrm{H}), 1.25-1.44(\mathrm{~m}, 26 \mathrm{H}), 0.87$ (t, $J=6.8 \mathrm{~Hz}, 3 \mathrm{H})$.

$S, S^{\prime}$-(5-(Hexadecyloxy)-1,3-phenylene)bis(methylene) diethanethioate (5b). Following the procedure described for $\mathbf{5 a}$, a mixture of $\mathbf{4 b}(4.10 \mathrm{~g}, 7.68 \mathrm{mmol})$ and potassium thioacetate $(2.3 \mathrm{~g}, 20 \mathrm{mmol})$ in ethanol $(300 \mathrm{~mL})$ was stirred under reflux to give $3.1 \mathrm{~g}(6.2 \mathrm{mmol})$ of $S, S^{\prime}-$ (5-(hexadecyloxy)-1,3-phenylene)bis(methylene) diethanethioate as a pale brown solid (82\% yield). ${ }^{1} \mathrm{H}$ NMR $\left(500 \mathrm{MHz}, \mathrm{CDCl}_{3}\right): \delta 6.75(\mathrm{~s}, 1 \mathrm{H}), 6.69(\mathrm{~s}, 2 \mathrm{H}), 4.03(\mathrm{~s}, 4 \mathrm{H}), 3.89$ (t, J = $6.8 \mathrm{~Hz}$, 2H), $2.33(\mathrm{~s}, 6 \mathrm{H}), 1.77(\mathrm{~m}, 2 \mathrm{H}), 1.25-1.44(\mathrm{~m}, 26 \mathrm{H}), 0.87$ (t, $J=6.8 \mathrm{~Hz}, 3 \mathrm{H})$.

(5-(Hexadecyloxy)-1,3-phenylene)dimethanethiol (HDPDT). Following the procedure described for PFPDT, a solution of $S, S^{\prime}$-(5-(hexadecyloxy)-1,3-phenylene)bis(methylene) diethanethioate $(3.1 \mathrm{~g}, 6.3 \mathrm{mmol})$ in a THF $(300 \mathrm{~mL})$ suspension of $\mathrm{LiAlH}_{4}(0.76 \mathrm{~g}, 20 \mathrm{mmol})$ was used to obtain the product. The crude product was purified by column chromatography (hexanes) to give $2.8 \mathrm{~g}(4.4 \mathrm{mmol})$ of (5-(2,5,8,11-tetraoxahexadecan-16-yloxy)-1,3phenylene)dimethanethiol as a white solid (70\% yield). $\left.{ }^{1} \mathrm{H} \mathrm{NMR} \mathrm{(500} \mathrm{MHz}, \mathrm{CDCl}_{3}\right): \delta 6.84$ (s, 1H), $6.75(\mathrm{~s}, 2 \mathrm{H}), 3.94(\mathrm{t}, J=6.3 \mathrm{~Hz}, 2 \mathrm{H}), 3.68(\mathrm{~d}, J=8.0 \mathrm{~Hz}, 4 \mathrm{H}), 1.76(\mathrm{~m}, 4 \mathrm{H}), 1.27-1.45(\mathrm{~m}$, $26 \mathrm{H}), 0.89(\mathrm{t}, J=6.3 \mathrm{~Hz}, 3 \mathrm{H}) .{ }^{13} \mathrm{C} \mathrm{NMR}\left(125 \mathrm{MHz}, \mathrm{CDCl}_{3}\right): \delta 159.72,142.99,119.88,112.97$, $68.11,32.06,29.84,29.75,29.72$, 29.53, 29.50, 29.40, 29.04, 26.18, 22.83, 14.28.

Synthesis of (5-(2,5,8,11-Tetraoxahexadecan-16-yloxy)-1,3-phenylene)dimethanethiol (OEGPDT). OEGPDT was prepared according to Scheme S1. The experimental details are provided in the following paragraphs.

5-(2,5,8,11-Tetraoxahexadecan-16-yloxy)-1,3-phenylene)dimethanol (3c). Following the procedure described for 3a, a mixture of $\mathrm{K}_{2} \mathrm{CO}_{3}(2.76 \mathrm{~g}, 20.0 \mathrm{mmol})$, bis(hydroxymethyl)phenol (2.00 g, $12.9 \mathrm{mmol})$ and 16-bromo-2,5,8,11-tetraoxahexadecane (3.60 g, $11.5 \mathrm{mmol})$ in acetonitrile $(250 \mathrm{~mL})$ was refluxed to give $3.00 \mathrm{~g}(7.70 \mathrm{mmol})$ of a clear oil product $(67 \%$ yield $)$. ${ }^{1} \mathrm{H}$ NMR $\left(500 \mathrm{MHz}, \mathrm{CDCl}_{3}\right): \delta 6.91(\mathrm{~s}, 1 \mathrm{H}), 6.83(\mathrm{~s}, 2 \mathrm{H}), 4.65(\mathrm{~s}, 4 \mathrm{H}), 3.96(\mathrm{t}, J=6.8 \mathrm{~Hz}, 2 \mathrm{H})$, $3.64(\mathrm{~m}, 8 \mathrm{H}), 3.57(\mathrm{~m}, 2 \mathrm{H}), 3.53(\mathrm{~m}, 2 \mathrm{H}), 3.47(\mathrm{t}, J=6.3 \mathrm{~Hz}, 2 \mathrm{H}), 3.36(\mathrm{~s}, 3 \mathrm{H}), 1.79(\mathrm{~m}, 2 \mathrm{H})$, $1.64(\mathrm{~m}, 2 \mathrm{H}), 1.51(\mathrm{~m}, 2 \mathrm{H})$. 
(5-(2,5,8,11-Tetraoxahexadecan-16-yloxy)-1,3-phenylene)bis(methylene) dimethanesulfonate $(\mathbf{4 c})$. Following the procedure described for $\mathbf{4 a}$, a mixture of $\mathbf{3 c}(3.00 \mathrm{~g}, 7.70 \mathrm{mmol})$ and triethylamine $(2.8 \mathrm{~mL}, 20 \mathrm{mmol})$ in $250 \mathrm{~mL}$ of THF was treated with methanesulfonyl chloride $(1.5 \mathrm{~mL}, 20 \mathrm{mmol})$ to give $4.00 \mathrm{~g}$ ( $7.38 \mathrm{mmol})$ of (5-(2,5,8,11-tetraoxahexadecan-16-yloxy)-1,3phenylene)bis(methylene) dimethanesulfonate (96\% yield). ${ }^{1} \mathrm{H}$ NMR $\left(500 \mathrm{MHz}, \mathrm{CDCl}_{3}\right): \delta 7.00$ (s, 1H), $6.94(\mathrm{~s}, 2 \mathrm{H}), 5.19(\mathrm{~s}, 4 \mathrm{H}), 3.96(\mathrm{t}, J=6.8 \mathrm{~Hz}, 2 \mathrm{H}), 3.64(\mathrm{~m}, 8 \mathrm{H}), 3.58(\mathrm{~m}, 2 \mathrm{H}), 3.54$ $(\mathrm{m}, 2 \mathrm{H}), 3.48(\mathrm{t}, J=6.3 \mathrm{~Hz}, 2 \mathrm{H}), 3.37(\mathrm{~s}, 3 \mathrm{H}), 2.97(\mathrm{~s}, 6 \mathrm{H}), 1.79(\mathrm{~m}, 2 \mathrm{H}), 1.64(\mathrm{~m}, 2 \mathrm{H}), 1.51$ $(\mathrm{m}, 2 \mathrm{H})$.

$S, S^{\prime}$-(5-(2,5,8,11-Tetraoxahexadecan-16-yloxy)-1,3-phenylene)bis(methylene) diethanethioate $(\mathbf{5 c})$. Following the procedure described for $\mathbf{5 a}$, a mixture of $\mathbf{4 c}(4.00 \mathrm{~g}, 7.38 \mathrm{mmol})$ and potassium thioacetate $(2.30 \mathrm{~g}, 20.1 \mathrm{mmol})$ in ethanol $(300 \mathrm{~mL})$ was stirred under reflux to give $2.60 \mathrm{~g}$ (5.18 mmol) of $S, S^{\prime}$-(5-(2,5,8,11-tetraoxahexadecan-16-yloxy)-1,3-phenylene)bis(methylene) diethanethioate as a brown oil (70\% yield). ${ }^{1} \mathrm{H} \mathrm{NMR}\left(500 \mathrm{MHz}, \mathrm{CDCl}_{3}\right): \delta 6.75(\mathrm{~s}, 1 \mathrm{H})$, $6.68(\mathrm{~s}, 2 \mathrm{H}), 4.03(\mathrm{~s}, 4 \mathrm{H}), 3.89(\mathrm{t}, J=6.3 \mathrm{~Hz}, 2 \mathrm{H}), 3.65(\mathrm{~m}, 8 \mathrm{H}), 3.58(\mathrm{~m}, 2 \mathrm{H}), 3.54(\mathrm{~m}, 2 \mathrm{H})$, $3.47(\mathrm{t}, J=6.8 \mathrm{~Hz}, 2 \mathrm{H}), 3.37(\mathrm{~s}, 3 \mathrm{H}), 2.34(\mathrm{~s}, 6 \mathrm{H}), 1.77(\mathrm{~m}, 2 \mathrm{H}), 1.64(\mathrm{~m}, 2 \mathrm{H}), 1.49(\mathrm{~m}, 2 \mathrm{H})$.

(5-(2,5,8,11-Tetraoxahexadecan-16-yloxy)-1,3-phenylene)dimethanethiol

(OEGPDT). Following the procedure described for PFPDT, A solution of $S, S^{\prime}-(5-(2,5,8,11$ tetraoxahexadecan-16-yloxy)-1,3-phenylene)bis(methylene) diethanethioate (2.60 g, $5.18 \mathrm{mmol})$ in a THF $(300 \mathrm{~mL})$ suspension of $\mathrm{LiAlH}_{4}(0.76 \mathrm{~g}, 20 \mathrm{mmol})$ was used to obtain the product. The crude product was purified by column chromatography (hexanes:ethyl acetate $=4: 6$ ) to give $1.24 \mathrm{~g}$ ( $2.97 \mathrm{mmol})$ of (5-(2,5,8,11-tetraoxahexadecan-16-yloxy)-1,3-phenylene)dimethanethiol as a clear oil (57\% yield). ${ }^{1} \mathrm{H}$ NMR $\left(500 \mathrm{MHz}, \mathrm{CDCl}_{3}\right): \delta 6.83(\mathrm{~s}, 1 \mathrm{H}), 6.72(\mathrm{~s}, 2 \mathrm{H}), 3.93(\mathrm{t}$, $J=6.3 \mathrm{~Hz}, 2 \mathrm{H}), 3.68(\mathrm{~d}, J=7.4 \mathrm{~Hz}, 4 \mathrm{H}), 3.64(\mathrm{~m}, 8 \mathrm{H}), 3.58(\mathrm{~m}, 2 \mathrm{H}), 3.54(\mathrm{~m}, 2 \mathrm{H}), 3.47(\mathrm{t}$, $J=6.3 \mathrm{~Hz}, 2 \mathrm{H}), 3.36(\mathrm{~s}, 3 \mathrm{H}), 1.78(\mathrm{~m}, 4 \mathrm{H}), 1.64(\mathrm{~m}, 2 \mathrm{H}), 1.51(\mathrm{~m}, 2 \mathrm{H}) .{ }^{13} \mathrm{C}$ NMR $(125 \mathrm{MHz}$, $\left.\mathrm{CDCl}_{3}\right): \delta 159.52,142.92,119.90,112.87,71.95,71.22,70.64,70.53,70.17,67.82,59.05,29.43$, $29.11,28.91$.

Synthesis of 9,9,10,10,11,11,12,12,13,13,14,14,15,15,16,16,16-Heptadecafluorohexa-decane1-thiol (PFT). PFT was prepared according to a procedure found in the literature. ${ }^{\mathrm{S} 1}{ }^{1} \mathrm{H}$ NMR $\left(500 \mathrm{MHz}, \mathrm{CDCl}_{3}\right): \delta 2.51(\mathrm{q}, J=7.4 \mathrm{~Hz}, 2 \mathrm{H}), 2.02(\mathrm{~m}, 2 \mathrm{H}), 1.58(\mathrm{~m}, 4 \mathrm{H}), 1.30-1.41(\mathrm{~m}, 9 \mathrm{H})$. ${ }^{13} \mathrm{C}$ NMR $\left(125 \mathrm{MHz}, \mathrm{CDCl}_{3}\right): \delta 34.01,31.06,30.88,30.71,29.17,29.08,28.88,28.30,24.62$, 20.10 .

Synthesis of 2,5,8,11-Tetraoxahexadecane-16-thiol (OEGT). OEGT was prepared according to a procedure found in the literature. ${ }^{\mathrm{S} 2}{ }^{1} \mathrm{H}$ NMR $\left(500 \mathrm{MHz}, \mathrm{CDCl}_{3}\right): \delta 3.62(\mathrm{~m}, 8 \mathrm{H}), 3.55(\mathrm{~m}$, $2 \mathrm{H}), 3.52(\mathrm{~m}, 2 \mathrm{H}), 3.43(\mathrm{t}, J=6.3 \mathrm{~Hz}, 2 \mathrm{H}), 3.35(\mathrm{~s}, 3 \mathrm{H}), 2.50(\mathrm{q}, J=6.8 \mathrm{~Hz}, 2 \mathrm{H}), 1.58(\mathrm{~m}, 4 \mathrm{H})$, $1.41(\mathrm{~m}, 2 \mathrm{H}), 1.31(\mathrm{t}, J=8.0 \mathrm{~Hz}, 1 \mathrm{H}) .{ }^{13} \mathrm{C} \mathrm{NMR}\left(125 \mathrm{MHz}, \mathrm{CDCl}_{3}\right): \delta 71.87,71.04,70.54$, $70.44,70.05,58.94,33.78,29.02,24.89,24.46$. 


\section{${ }^{1} \mathrm{H}$ and ${ }^{13} \mathrm{C}$ NMR Spectra.}

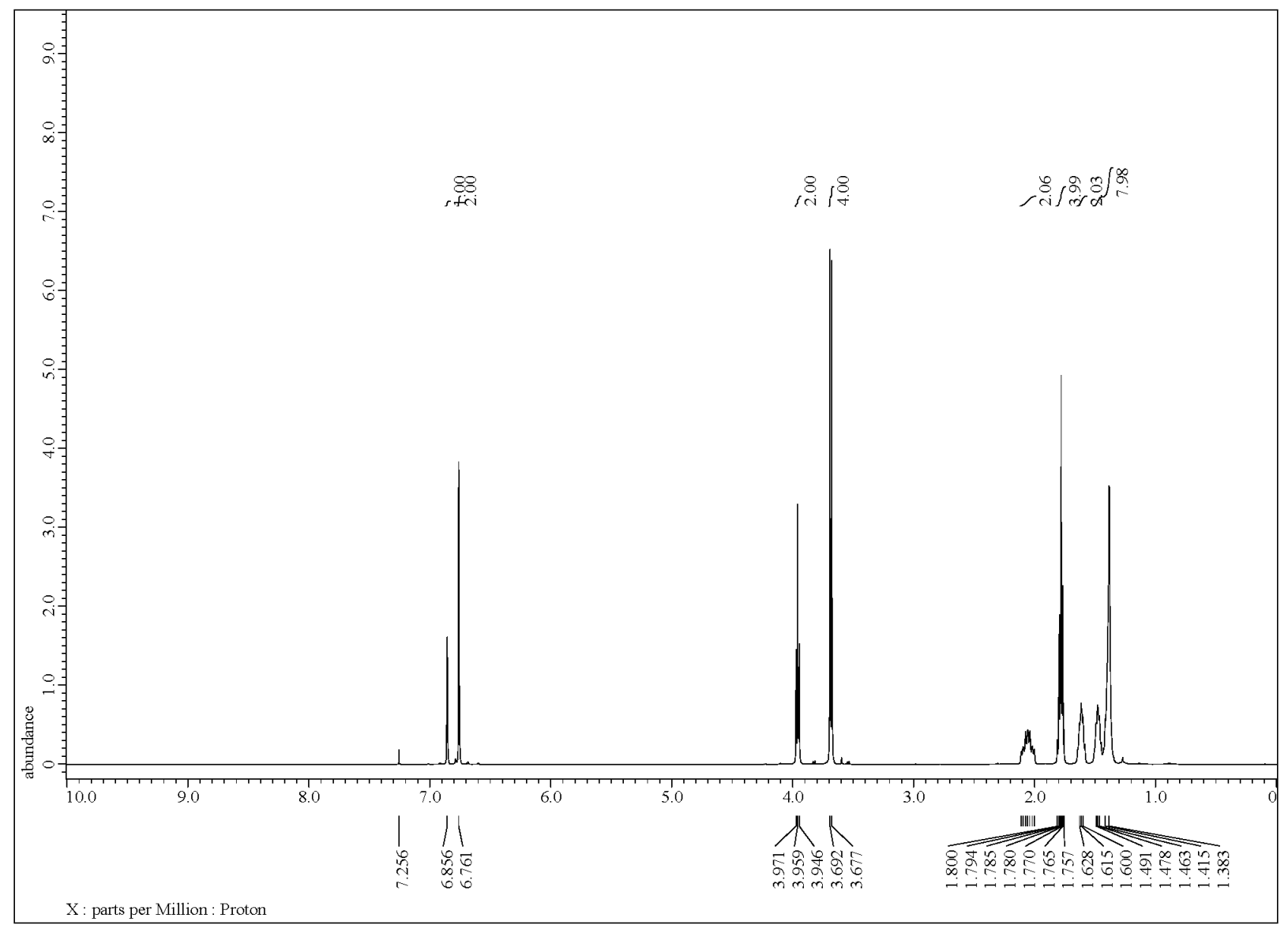

Figure S1. $\quad{ }^{1} \mathrm{H}$ NMR spectrum of $(5-(9,9,10,10,11,11,12,12,13,13,14,14,15,15,16,16,16-$ heptadecafluorohexadecyloxy)-1,3-phenylene)dimethanethiol (PFPDT). 


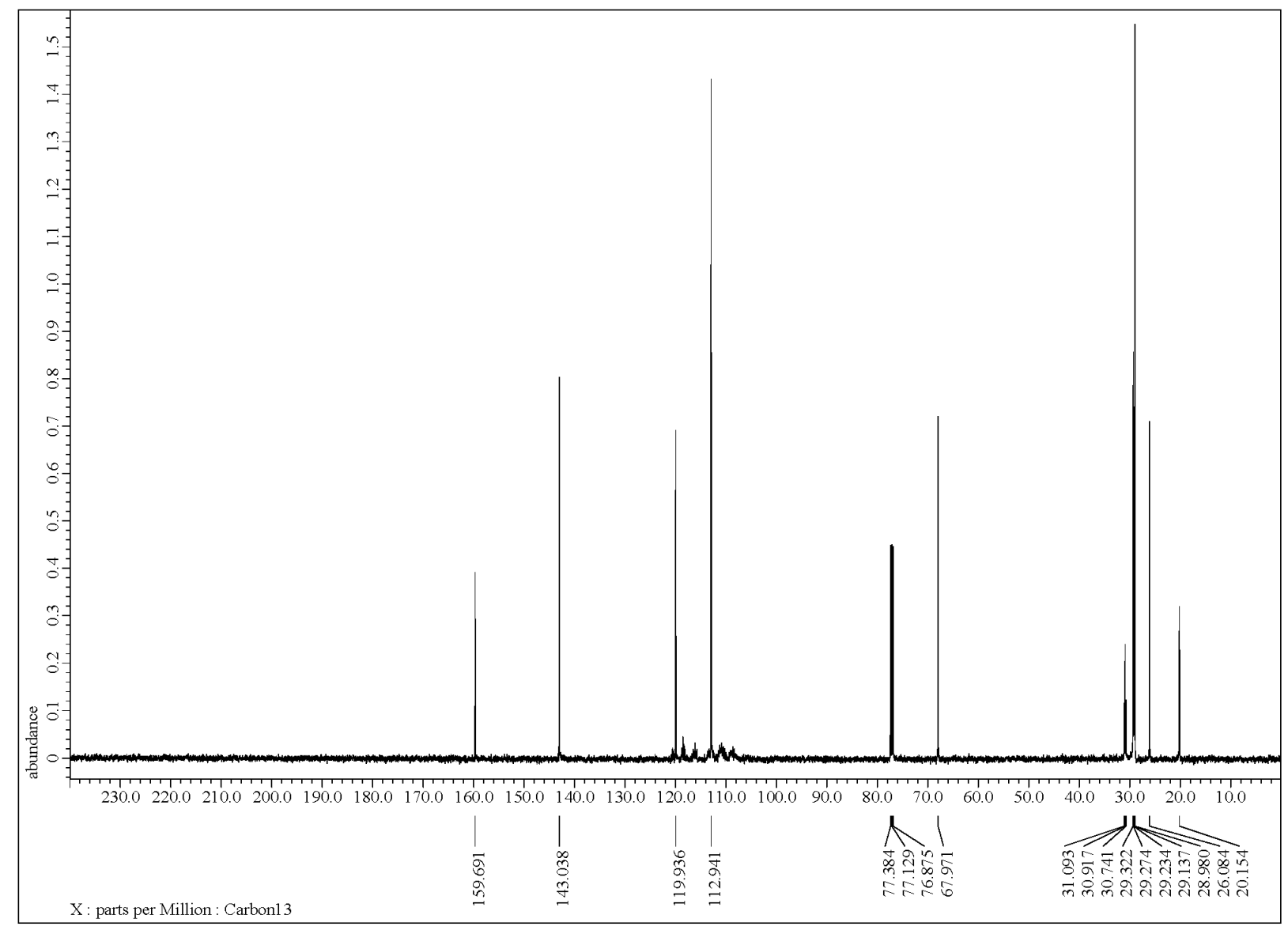

Figure S2. ${ }^{13} \mathrm{C}$ NMR spectrum of $(5-(9,9,10,10,11,11,12,12,13,13,14,14,15,15,16,16,16$-heptadecafluorohexadecyloxy)-1,3-phenylene)dimethanethiol (PFPDT). 


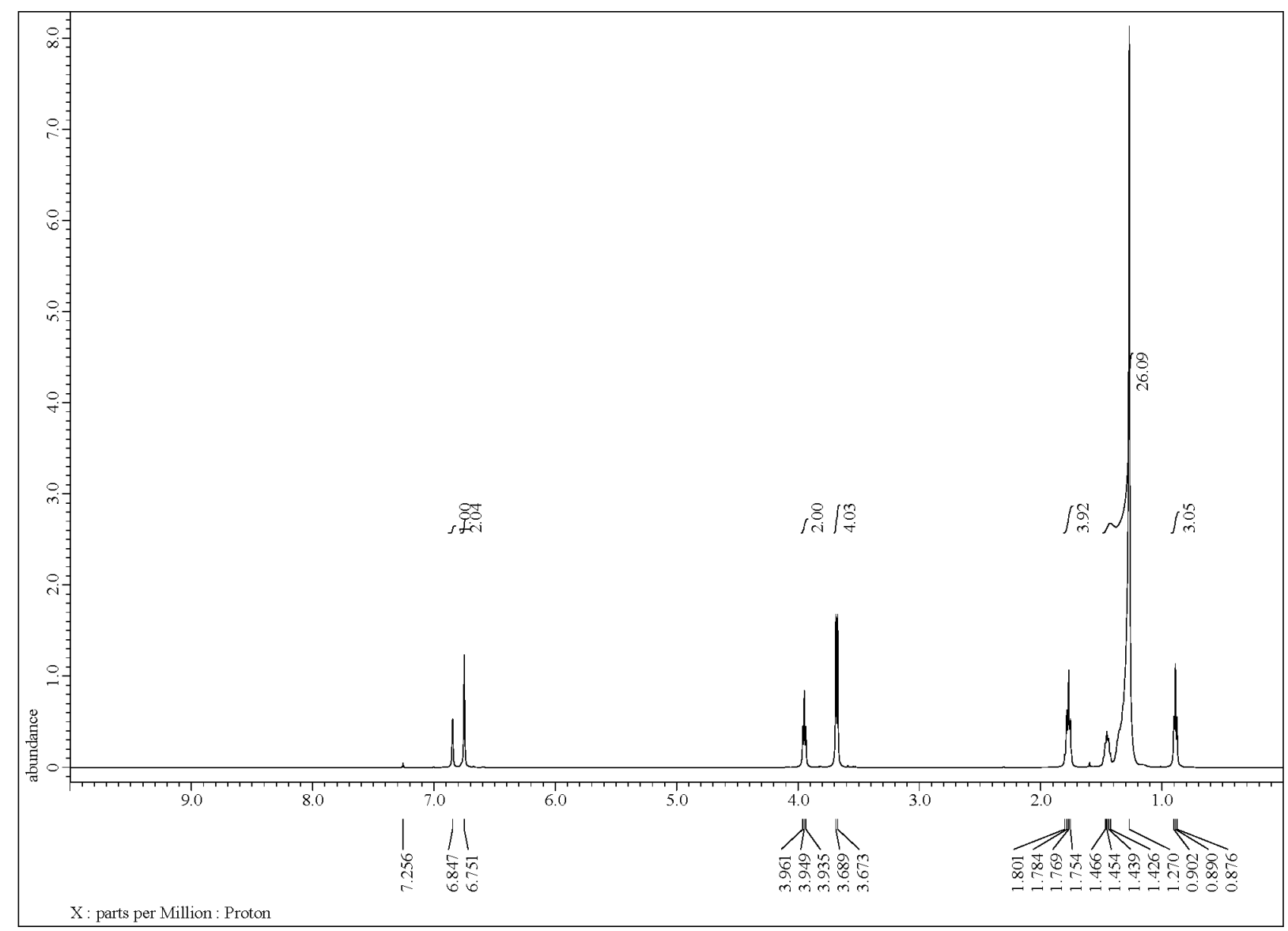

Figure S3. ${ }^{1} \mathrm{H}$ NMR spectrum of (5-(hexadecyloxy)-1,3-phenylene)dimethanethiol (HDPDT). 


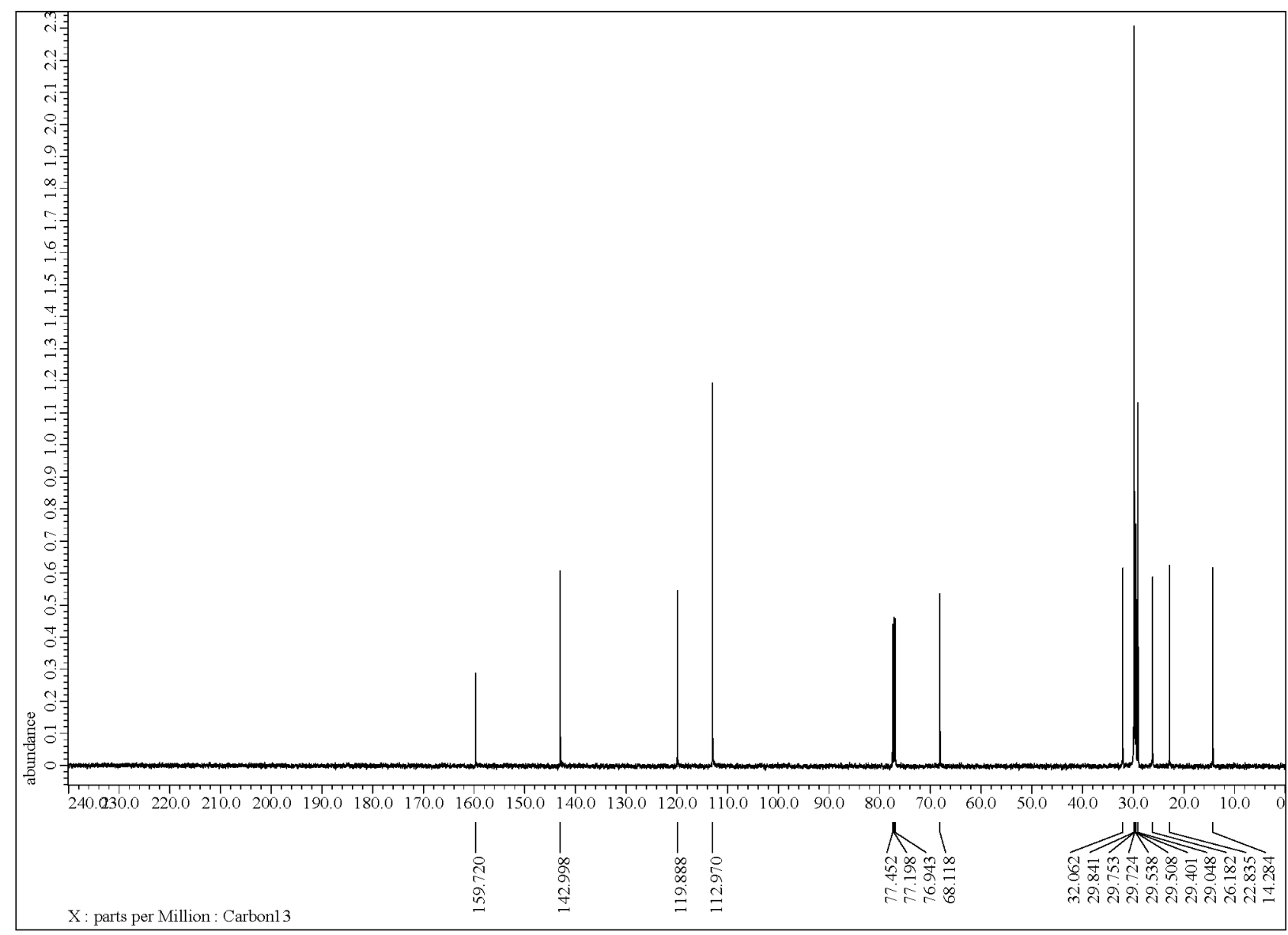

Figure S4. ${ }^{13} \mathrm{C}$ NMR spectrum of (5-(hexadecyloxy)-1,3-phenylene)dimethanethiol (HDPDT). 


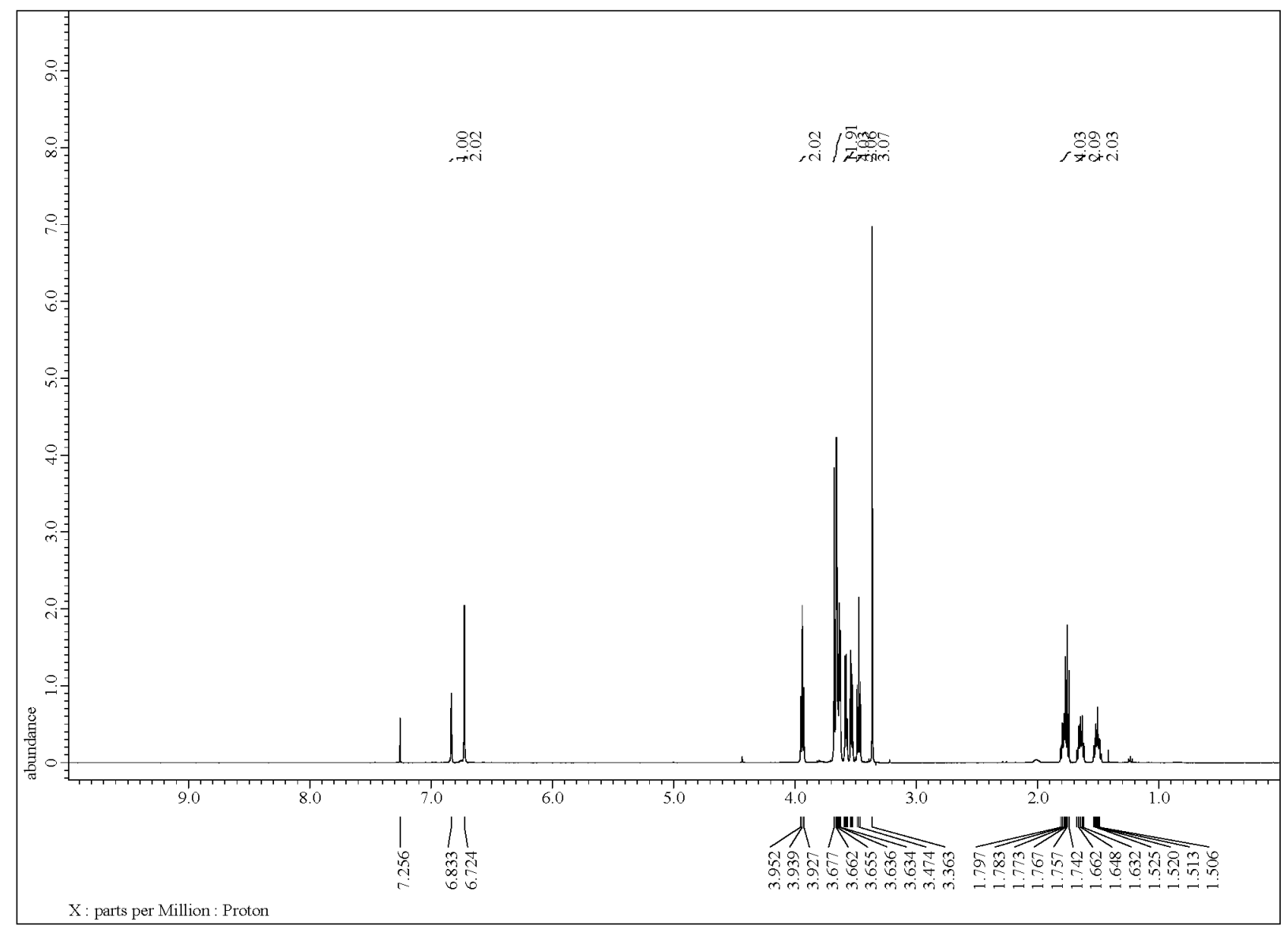

Figure S5. ${ }^{1} \mathrm{H}$ NMR spectrum of (5-(2,5,8,11-tetraoxahexadecan-16-yloxy)-1,3-phenylene)dimethanethiol (OEGPDT). 


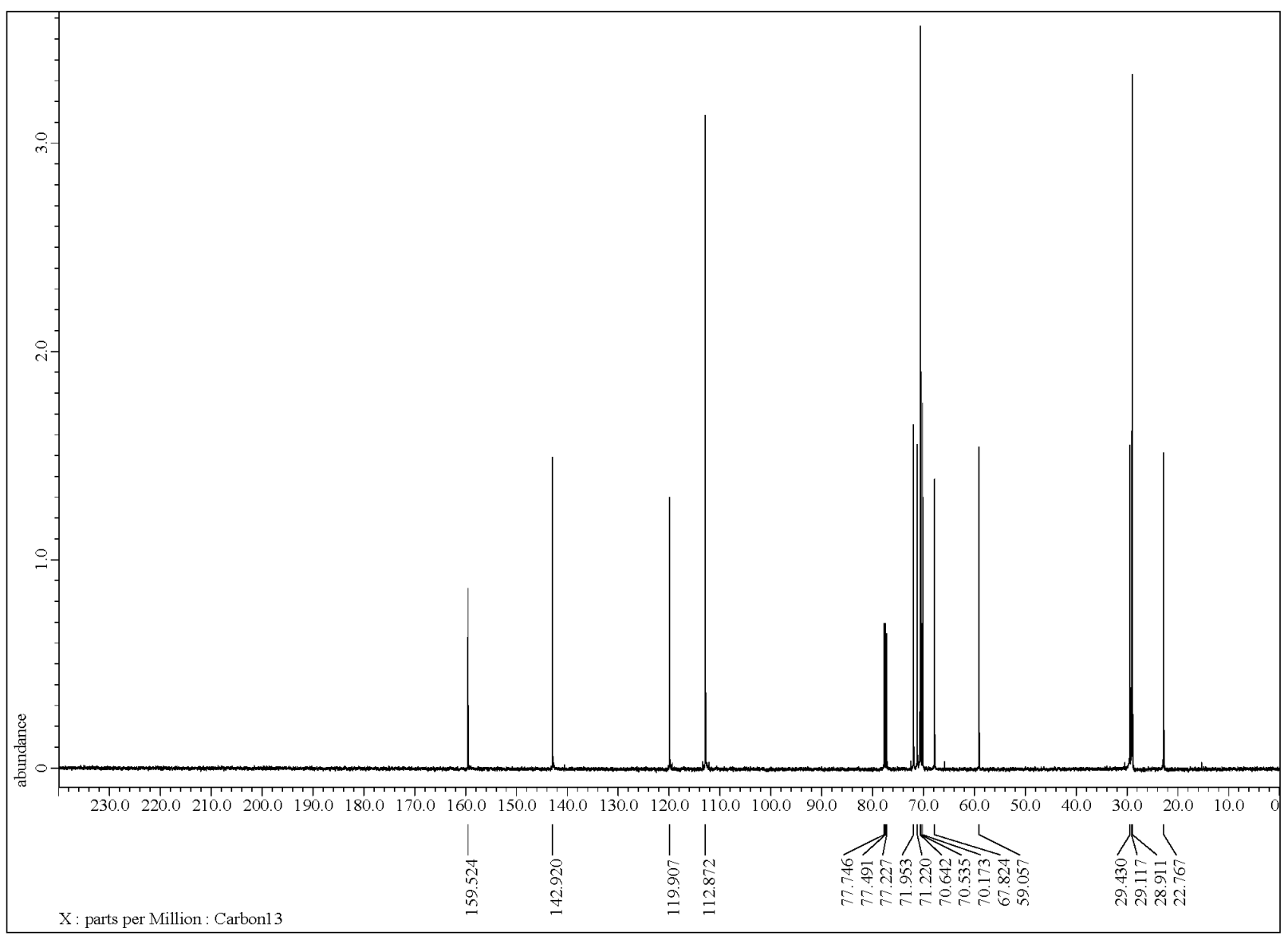

Figure S6. ${ }^{13} \mathrm{C}$ NMR spectrum of (5-(2,5,8,11-tetraoxahexadecan-16-yloxy)-1,3-phenylene)dimethanethiol (OEGPDT). 
Preparation of Evaporated Gold Surfaces. Gold pellets (99.999\%) were purchased from Kamis Inc., chromium-coated rods from Kurt J. Lesker Co., and polished single crystal silicon(100) wafers were purchased from University Wafer. A thermal evaporator was used to prepare the flat gold substrates. Gold films (thickness: $\sim 1000 \AA$, rate of evaporation: $\sim 0.5 \AA / \mathrm{sec}$ ) were formed on chromium-coated (thickness: $\sim 100 \AA$, rate of evaporation: $\sim 0.5 \AA / \mathrm{sec}$ ) Si wafers under ultrahigh vacuum. The gold-coated wafers were then cut into slides $(1 \times 4 \mathrm{~cm})$, rinsed with ethanol, and dried with ultrapure nitrogen gas before formation of the monolayer films.

Preparation of SAMs on Evaporated Gold Surfaces. Organic thin films were prepared by immersing slides $(1 \times 4 \mathrm{~cm})$ of freshly prepared gold-coated silicon wafers in ethanolic solutions containing the binary mixture of thiols. The surfactant ratios were varied while the overall thiol concentration was maintained at $1.0 \mathrm{mM}$. For each SAM series (monodentate and bidentate), nine monolayers were prepared with the following development solution ratios (the "solution composition", where A/B/C are PFT/HDT/OEGT and PFPDT/HDPDT/OEGPDT):

$100 \% \mathrm{~A}$

$75 \%$ A with $25 \% \mathrm{~B}$

$50 \%$ A with $50 \%$ B

$25 \%$ A with $75 \%$ B

$100 \% \mathrm{~B}$

$75 \%$ B with $25 \% \mathrm{C}$

$50 \%$ B with $50 \% \mathrm{C}$

$25 \%$ B with $75 \%$ C

$100 \% \mathrm{C}$

The glass vials containing the solutions were previously cleaned with piranha solution (3:1 mixture of concentrated $\mathrm{H}_{2} \mathrm{SO}_{4} / 30 \mathrm{wt} \% \mathrm{H}_{2} \mathrm{O}_{2}$ ) and rinsed thoroughly with copious amounts of deionized water and finally with ethanol. Caution: piranha solution is highly corrosive, should never be stored, and should be handled with extreme caution! We also prepared SAMs using normal octadecanethiol (C18SH) as a reference SAM since octadecanethiol provides welldefined organic thin films on flat gold surfaces. All slides were incubated for $48 \mathrm{~h}$, rinsed thoroughly with THF and ethanol, and then dried with a gentle stream of ultrapure nitrogen gas before analysis.

Characterization of SAMs on Evaporated Gold Surfaces. The data for the contact angle measurements, ellipsometric film thicknesses, X-ray photoelectron spectroscopy (XPS) spectra, and polarization modulation infrared reflection-absorption spectroscopy (PM-IRRAS) spectra were obtained with the same instruments under the same conditions found in a previous study. ${ }^{\mathrm{S3}}$ 
Additional Wettability Data. The hydrophobicity and hydrophilicity of the films generated from mixtures of the perfluoro-, $n$-alkyl-, and oligo(ethylene glycol)-terminated adsorbates were evaluated by collecting both the advancing contact angles $\left(\theta_{\mathrm{a}}\right)$ and receding contact angles $\left(\theta_{\mathrm{r}}\right)$ for water. These data, along with the hysteresis values $\left(\theta_{\mathrm{a}}-\theta_{\mathrm{r}}\right)$ for all of the SAMs prepared for this study, are provided in Table S1.

Table S1. Advancing Contact Angles $\left(\theta_{\mathrm{a}}\right)$, Receding Contact Angles $\left(\theta_{\mathrm{r}}\right)$, and Hysteresis Values $\left(\theta_{\mathrm{a}}-\theta_{\mathrm{r}}\right)$ for Water on Both Series of Mixed SAMs

\begin{tabular}{|c|c|c|c|c|c|c|c|}
\hline \multirow[b]{2}{*}{ Mixed SAM } & \multicolumn{3}{|c|}{ contact angle $^{a}$} & \multirow[b]{2}{*}{ Mixed SAM } & \multicolumn{3}{|c|}{ contact angle $\mathrm{a}^{\mathrm{a}}$} \\
\hline & $\begin{array}{l}\text { advancing } \\
\left(\theta_{\mathrm{a}}\right)\end{array}$ & $\begin{array}{l}\text { receding } \\
\left(\theta_{\mathrm{r}}\right)\end{array}$ & $\begin{array}{l}\text { hysteresis } \\
\left(\theta_{\mathrm{a}}-\theta_{\mathrm{r}}\right)\end{array}$ & & $\begin{array}{l}\text { advancing } \\
\left(\theta_{\mathrm{a}}\right)\end{array}$ & $\begin{array}{c}\text { receding } \\
\left(\theta_{\mathrm{r}}\right)\end{array}$ & $\begin{array}{c}\text { hysteresis } \\
\left(\theta_{\mathrm{a}}-\theta_{\mathrm{r}}\right)\end{array}$ \\
\hline PFT (1.00) & $120^{\circ}$ & $102^{\circ}$ & $8^{\circ}$ & PFPDT (1.00) & $121^{\circ}$ & $104^{\circ}$ & $7^{\circ}$ \\
\hline PFT (0.75) & $115^{\circ}$ & $102^{\circ}$ & $13^{\circ}$ & PFPDT (0.75) & $118^{\circ}$ & $104^{\circ}$ & $14^{\circ}$ \\
\hline PFT (0.50) & $112^{\circ}$ & $102^{\circ}$ & $10^{\circ}$ & PFPDT (0.50) & $116^{\circ}$ & $104^{\circ}$ & $12^{\circ}$ \\
\hline PFT (0.25) & $109^{\circ}$ & $103^{\circ}$ & $6^{\circ}$ & PFPDT (0.25) & $112^{\circ}$ & $104^{\circ}$ & $8^{\circ}$ \\
\hline HDT & $108^{\circ}$ & $100^{\circ}$ & $8^{\circ}$ & HDPDT & $108^{\circ}$ & $102^{\circ}$ & $6^{\circ}$ \\
\hline OEGT (0.25) & $107^{\circ}$ & $99^{\circ}$ & $8^{\circ}$ & OEGPDT (0.25) & $103^{\circ}$ & $95^{\circ}$ & $8^{\circ}$ \\
\hline OEGT (0.50) & $107^{\circ}$ & $99^{\circ}$ & $8^{\circ}$ & OEGPDT (0.50) & $96^{\circ}$ & $91^{\circ}$ & $5^{\circ}$ \\
\hline OEGT (0.75) & $105^{\circ}$ & $98^{\circ}$ & $7^{\circ}$ & OEGPDT (0.75) & $80^{\circ}$ & $72^{\circ}$ & $8^{\circ}$ \\
\hline OEGT (1.00) & $52^{\circ}$ & $47^{\circ}$ & $5^{\circ}$ & OEGPDT (1.00) & $52^{\circ}$ & $46^{\circ}$ & $6^{\circ}$ \\
\hline
\end{tabular}

${ }^{\mathrm{a}}$ The reproducibilities of the contact angles were within $\pm 2^{\circ}$. 
(A)

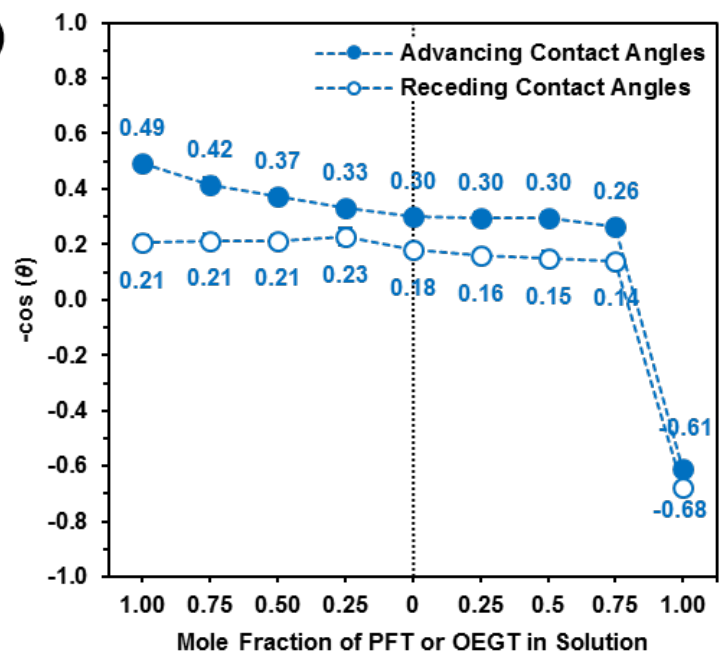

(B)

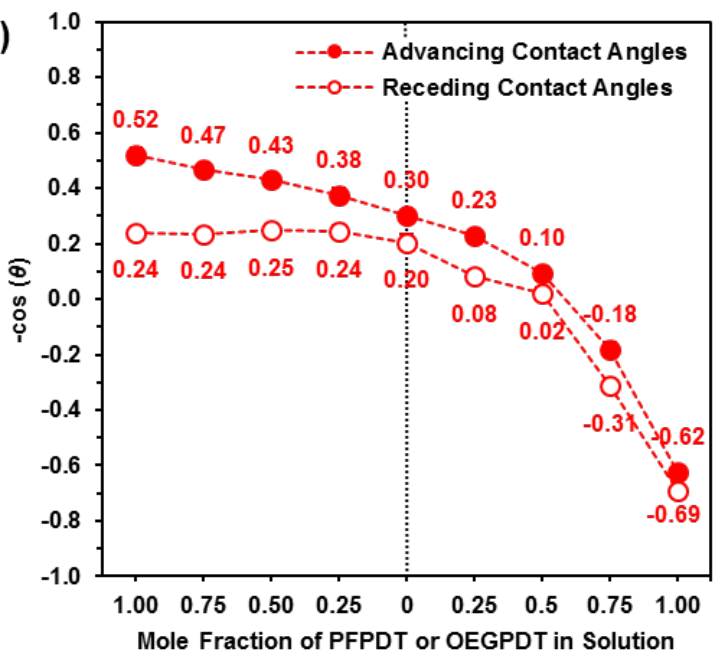

Figure S7. Cos $(\theta)$ values for advancing and receding contact angles of water on the mixed SAM series derived from (A) the monodentate alkanethiols (PFT, HDT, and OEGT), and (B) the bidentate alkanethiols (PFPDT, HDPDT, and OEGPDT) in ethanol. For each series, $\cos (\theta)$ values for the single-component $n$-alkyl-terminated SAM corresponds to mole fraction 0 in the plot. Error bars that are not visible fall within the symbols. 
(A)

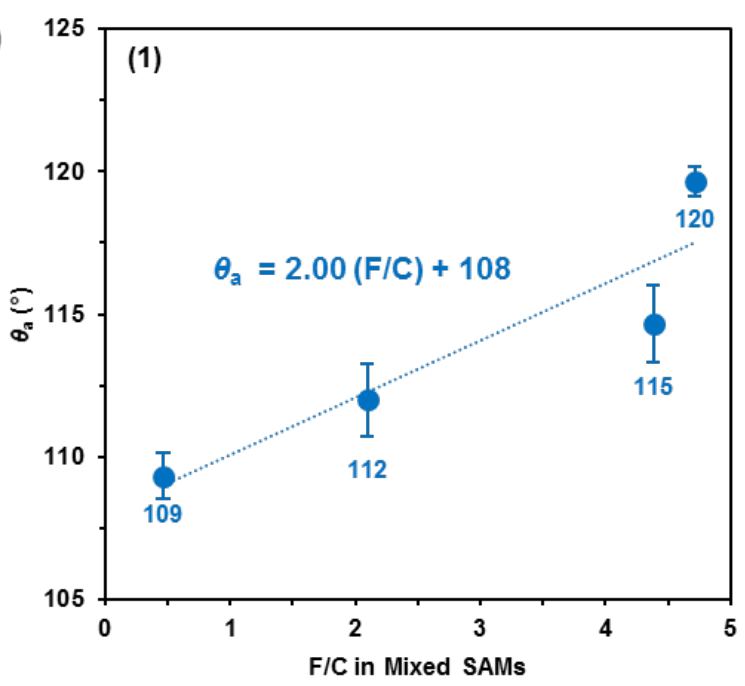

(B)

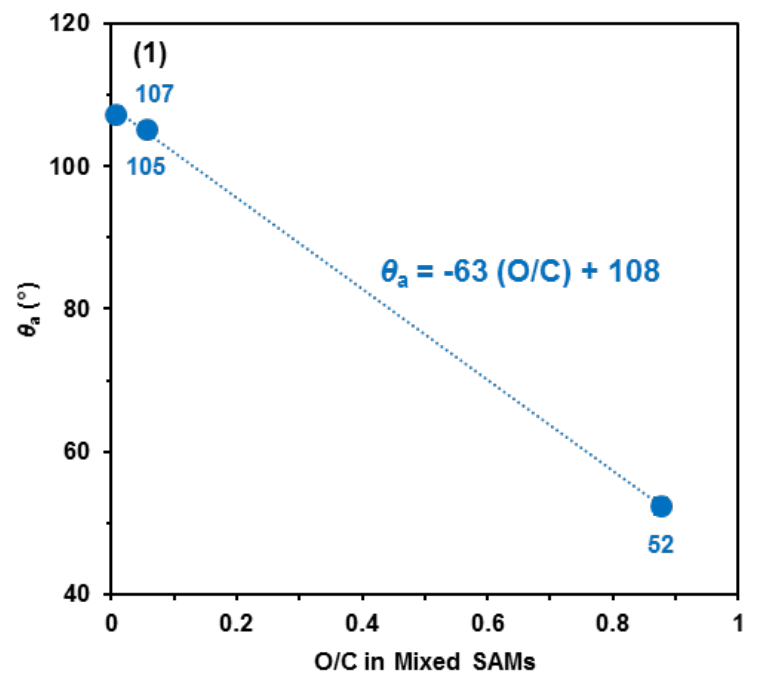

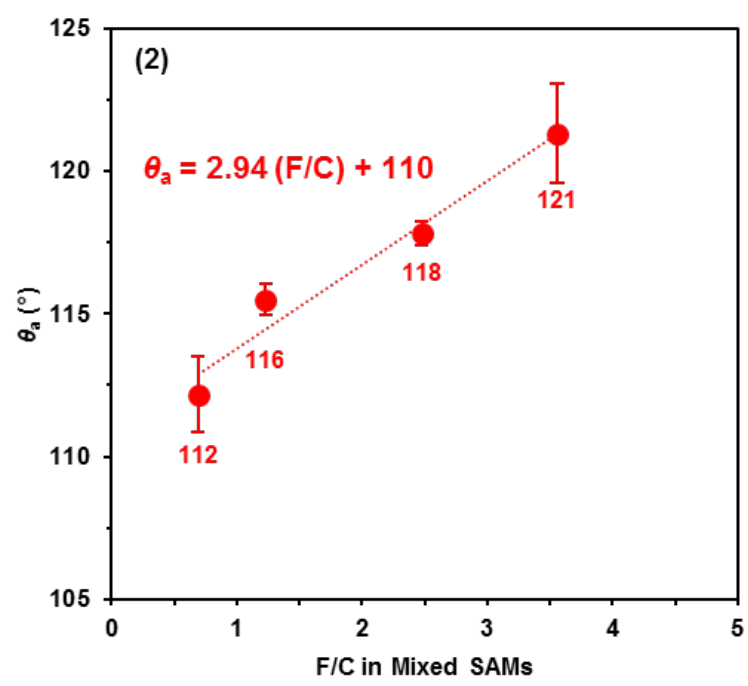

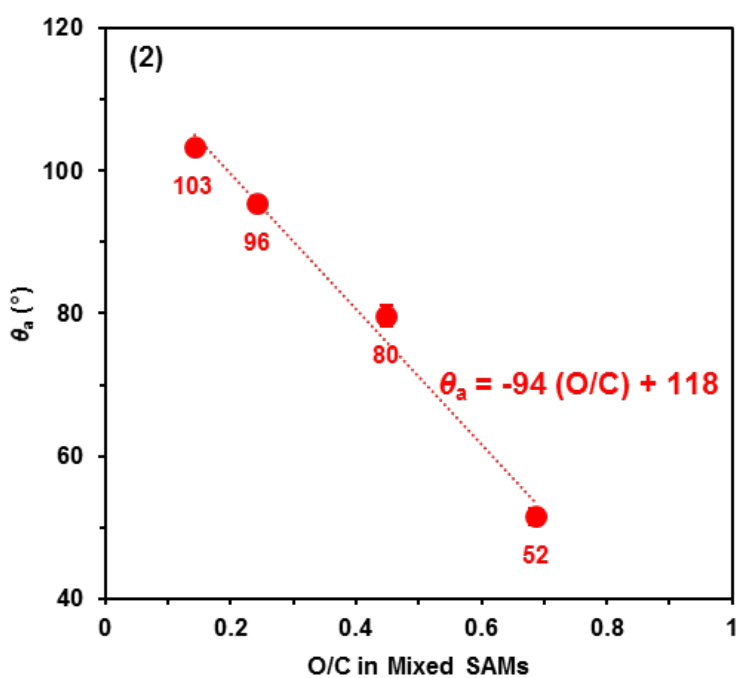

Figure S8. Advancing contact angles $\left(\theta_{\mathrm{a}}\right)$ of water on the mixed SAM as a function of (A) the fluorine-to-carbon $(\mathrm{F} / \mathrm{C})$ ratios and $(\mathrm{B})$ the oxygen-to-carbon $(\mathrm{O} / \mathrm{C})$ ratios for the SAMs derived from (1) the monodentate alkanethiols (PFT, HDT, and OEGT), and (2) the bidentate alkanethiols (PFPDT, HDPDT, and OEGPDT) in ethanol. Error bars indicate the standard deviation for the data. 


\section{References}

(S1) Graupe, M.; Koini, T.; Wang, V. Y.; Nassif, G. M.; Colorado, R., Jr.; Villazana, R. J.; Dong, H.; Miura, Y. F.; Shmakova, O. E.; Lee, T. R. Terminally Perfluorinated LongChain Alkanethiols. J. Fluorine Chem. 1999, 93, 107-115.

(S2) Drechsler, U.; Fischer, N. O.; Frankamp, B. L.; Rotello, V. M. Highly Efficient Biocatalyst via Covalent Immobilization of Candida rugosa Lipase on Ethylene Glycol-Modified Gold-Silica Nanocomposites. Adv. Mater. 2004, 29, 271-274.

(S3) Lee, H. J.; Jamison, A. C.; Yuan, Y.; Li, C.-H.; Rittikulsittichai, S.; Rusakova, I.; Lee, T. R. Robust Carboxylic Acid-Terminated Organic Thin Films and Nanoparticle Protectants Generated from Bidentate Alkanethiols. Langmuir 2013, 29, 10432-10439. 